\title{
COMMENTS TO THE AMINO ACID SEQUENCE OF YEAST GLYCERALDEHYDE-3-PHOSPHATE DEHYDROGENASE
}

\author{
by \\ STEPHEN BAYNE ${ }^{1}$, BRIAN MARTIN ${ }^{2}$ and IB SVENDSEN \\ Department of Chemistry, Carlsberg Laboratory, \\ Gamle Carlsberg Vej 10, DK-2500 CopenhagenValby \\ 'Present address: Fachbereich Biologie, Postfach 5560, D-7750 Konstanz, West Germany \\ 2Present address: Max Planck Institut für Ernährungsphysiologie, D-4600 Dortmund, West Germany
}

Keywords: Yeast glyceraldehyde-3-phosphate dehydrogenase, primary structure corrections, Edman degradation

The amino acid sequence of yeast glyceraldehyde-3-phosphate dehydrogenase has been reinvestigated by automated Edman degradation for those parts where discrepancies between previously published sequences are noted. In all instances our data confirmed those predicted from the corresponding gene nucleotide sequence.

\section{INTRODUCTION}

Glyceraldehyde-3-phosphate dehydrogenase, which catalyses reversibly the interconversion of D-glyceraldehyde-3-phosphate and 1,3-diphosphoglycerate, is a key enzyme in the metabolism of many organisms. The reaction mechanism and X-ray structure of this enzyme have been extensively investigated and the amino acid sequences of the pig, lobster and yeast enzymes elucidated $(1,4)$.

Three slightly different amino acid sequences have been reported for yeast glyceraldehyde-3- phosphate dehydrogenase (GAPDH) $(1,2,4)$. Two of these sequences were determined by dansyl-Edman analysis of the GAPDH polypeptide chain $(1,4)$. Although both these sequences were reported by the same group, several possibly significant differences in the primary structure can be noted. The third sequence, which was determined from the gene nucleotide sequence (2), is again different in several locations from either of those previously published.

The present paper reports corrections to the amino acid sequence of GAPDH as found by

Abbreviations: DPCC = diphenylcarbonylchloride, GAPDH = glyceraldehyde-3-phosphate dehydrogenase, Polybrene $=$ hexadimethrine bromide. 
automatic Edman degradation of peptides obtained from chemical and enzymatic cleavage. The resulting primary structure obtained is in good agreement with that predicted from the gene sequence.

\section{MATERIALS AND METHODS}

\subsection{Materials}

GAPDH was a commercial product of Boehringer, Mannheim. Isozyme III was isolated from this preparation as described by STaLlCUP et al. (7); however, DE-52 cellulose (Whatman Biochemicals, Maidstone, England) was used instead of DEAE-Sephadex A-25. The isolated isozyme III was found to be pure by electrophoresis in $3.75 \%$. polyacrylamide gels. The enzyme was then concentrated and subsequently desalted on a column of Biogel P-2 (Biorad, Richmond, California) prior to freeze-drying. This freeze-dried enzyme was used in all further chemical and enzymatic cleavages.

DPCC-trypsin and o-iodosobenzoic acid were supplied by Sigma Chemicals, St. Louis, Mo., USA. Highly purified carboxypeptidase Y was a commercial preparation from United Breweries, Inc. Suppliers for the chemicals used in the sequencer analyses have been described previously (3). All other chemicals were analytical grade and distilled water was used throughout.

\subsection{Sequence and amino acid analysis}

Amino acid sequence analysis using the Beckman $890 \mathrm{C}$ sequencer was carried out as described by Johansen et al. (3). Polybrene was used in all instances when peptides were sequenced. The dummy run was performed once to clear the Polybrene of impurities. The PTHamino acids were identified by high performance liquid chromatography as previously described (3) with sodium acetate $(\mathrm{pH}$ 4.03):methanol:acetonitrile $(9: 1: 1)$ as solvent $A$, and methanol:water $(9: 1)$ as solvent $B$. The amino acid compositions of peptides were obtained by hydrolysis for 24 hours at $110^{\circ} \mathrm{C}$ and subsequent analysis using a Durrum D-500 amino acid analyser. No corrections were made for degradation of threonine or serine or for incomplete hydrolysis of valine and isoleucine.

\subsection{Chemical and enzymatic cleavage of GAPDH}

\subsubsection{Chemical cleavage}

2.3.1.1.

Cyanogen bromide cleavage of GAPDH was performed as described previously for superoxide dismutase (3). The cleavage products, after removal of the excess reagent with a stream of nitrogen gas, were then directly fractionated on a column of Biogel P-10, equilibrated and eluted with $30 \%$ acetic acid, or they were diluted with water and freeze-dried, prior to tryptic digestion (see below). Peptide fractions from the column chromatography were pooled after monitoring the absorption at $280 \mathrm{~nm}$, freeze-dried and redissolved in a minimal volume of $30 \%$ acetic acid. Sequence studies were performed on these pools.

\subsubsection{2}

GAPDH $(10 \mathrm{mg})$ was dissolved in $70 \%$ formic acid $(1 \mathrm{ml})$ and incubated in the dark at $40^{\circ} \mathrm{C}$ for several days (5). A sample of this solution was applied directly to the sequencer.

\subsubsection{3.}

Cleavage of tryptophanyl bonds was effected by the use of o-iodosobenzoic acid (6). GAPDH (20 $\mathrm{mg}$ ) was dissolved in $2.5 \mathrm{ml} 4 \mathrm{M}$-guanidinium hydrochloride- $12 \mathrm{~mm}$-potassium phosphate buffer, $\mathrm{pH}$ 6. $50 \mathrm{mg}$ o-iodosobenzoic acid were added and the resulting white suspension stirred for $30 \mathrm{~min}$ at room temperature. Glacial acetic acid $(9 \mathrm{ml})$ was slowly added to the stirred suspension which gradually clarified. The homogeneous solution was left at room temperature in the dark for 24 hours and then freeze-dried. The resulting crystals were dissolved in $2 \mathrm{ml} 30 \%$ acetic acid (any precipitate of benzoic acid was removed by centrifugation) and applied to a Biogel P-10 column equilibrated and eluted with $30 \%$ acetic acid as described above. Appropriate fractions absorbing at $280 \mathrm{~nm}$ were pooled, freeze-dried and redissolved in a minimal volume of $30 \%$ acetic acid prior to sequence analysis. 


\subsubsection{Proteolytic cleavage}

\subsubsection{1.}

Tryptic digestion, as described previously for superoxide dismutase (3), was carried out on the cyanogen bromide cleavage products of GAPDH. In the present report an enzyme to substrate ratio of 1 to 50 was employed. After 6 hours digestion, the clear solution was freezedried prior to fractionation on a column of Biogel $\mathrm{P}-10$ as described above in section 2.3.1.1.

\subsubsection{2.}

Carboxypeptidase $Y$ digestion was also carried out on GAPDH, essentially as described for superoxide dismutase (3). The products were analysed directly by amino acid analysis.

\section{RESULTS AND DISCUSSION}

Our strategy for the elucidation of the inconsistencies in the GAPDH primary sequence involved a combination of both enzymatic and

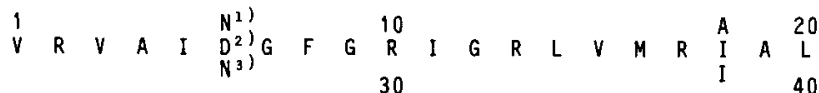

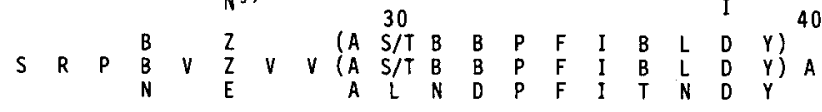

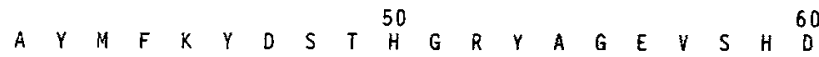

$$
\begin{aligned}
& \begin{array}{lllllllllllllllllllllllllllllll}
70 & & K & H & I & I & V & D & G & K & K & I & A & T & Y & Q & E & R & D & P & A
\end{array}
\end{aligned}
$$

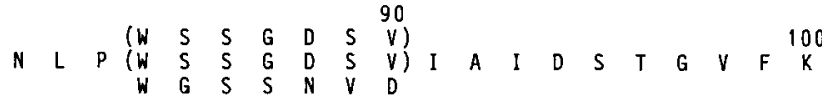

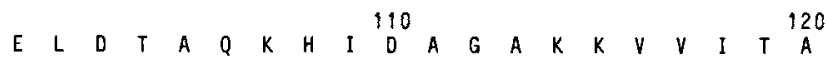

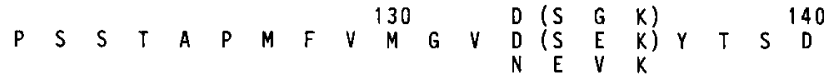

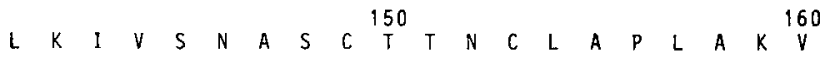

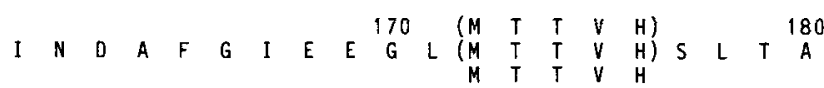

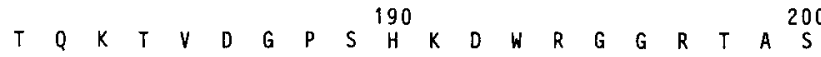

$$
\begin{aligned}
& \begin{array}{lllllllllllllllllllllll}
220 & N & I & I & P & S & S & T & G & A & A & K & A & V & G & K & V & L & P & E
\end{array}
\end{aligned}
$$

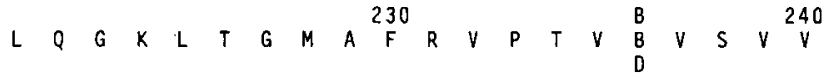

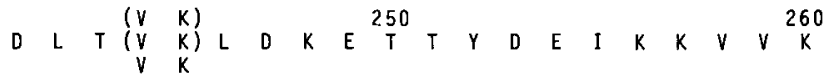

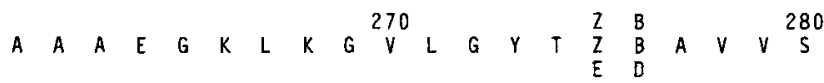

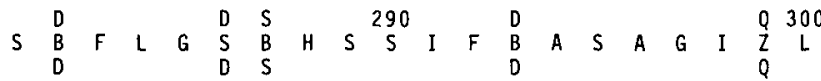

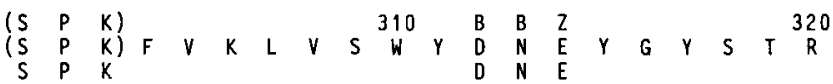

$$
\begin{aligned}
& V \quad V D L V E H \underset{I}{V / I} A \stackrel{330}{K} A
\end{aligned}
$$

Figure 1. Previously published sequences of GAPDH.

1) From reference 1. 2) From reference 4. 3) From reference 2. $A=A l a, B=A s x, C=C y s, D=A s p, E$ $=$ Glu, $\mathrm{F}=$ Phe, $\mathrm{G}=$ Gly, $\mathrm{H}=$ His, $\mathrm{I}=$ Ile, $\mathrm{K}=$ Lys, $\mathrm{L}=$ Leu, $\mathrm{M}=$ Met, $\mathrm{N}=$ Asn, $\mathrm{P}=$ Pro, $\mathrm{Q}=$ Gln, $\mathrm{R}=\operatorname{Arg}, \mathrm{S}=\operatorname{Ser}, \mathrm{T}=\mathrm{Thr}, \mathrm{V}=\mathrm{Val}, \mathrm{Y}=\mathrm{Tyr}, \mathrm{Z}=\mathrm{Glx}$. 
chemical cleavages at specific amino acid residues in the polypeptide chain. Fractionation of the resulting peptides and subsequent sequence analysis allowed corrections to the primary structure to be suggested.

The three previously published sequences of GAPDH are presented in Figure 1. Where no differences among these three sequences occur, a single amino acid residue is noted. Where any difference among the three reported sequences occurs, all three suggested variations are noted. The residues in parenthesis signify those residues in the yeast enzyme which give a "best fit" agreement with the corresponding residues and sequence in the pig and lobster enzymes (1).

As can be seen in Figure 1, the major differences among the three listed sequences occur between those determined from the amino acid sequence analysis and that predicted from the gene sequence. More specifically, differences exist in segments comprising residues 29 to 39 , residues 84 to 90 and residues 133 to 135 . The other differences concern mainly the substitution of an acidic for an amido residue or v.v. The possibility exists that the protein used for the sequence structure determinations $(1,4)$ was a mixture of the isozymes present in yeast.

The choice of reagents for specific chemical cleavages in proteins is limited. For GAPDH, it is fortunate that many of the inconsistent sequences occur after methionine and tryptophan peptide bonds which have previously been shown to be susceptible to specific chemical cleavage.
Our initial procedure was to sequence the unmodified protein from the $\mathrm{N}$-terminus which allowed identification of residue 6 as asparagine and residue 18 as isoleucine. After residue 24, which was an asparagine, overlaps and incomplete splitting occurred which did not allow unequivocal identification of residues 26 to 37 but nevertheless permitted a tentative sequence to be advanced

-Glu-Val-Val-Ala-Leu-Asn-Asp-Pro-Phe-IleThr-Asn-

Incubation of proteins in $70 \%$ formic acid has been shown to result in cleavage of only aspartic acid - proline peptide bonds (5). Since the published sequences of GAPDH show two such peptide sequences (residues 32-33 and residues 78-79), GAPDH was treated with $70 \%$ formic acid. No fractionation of the peptides was carried out before sequence analysis since only three peptides should be obtained. Figure $2(\mathrm{~A})$, which compares the sequences of the three possible peptides, indicates that only in cycles 1,7 and 9 should the same amino acids be obtained in more than one sequence. Figure 2 (B) indicates the sequences which were found and these agree with those predicted by the gene structure. It should be noted that a similar formic acid treatment of one of the other isozymes isolated during present studies in yeast produced sequences similar to those reported in references 1 and 4. This would indicate that another isozyme than that which was used in the present studies was employed in earlier primary structure studies.

\begin{tabular}{|c|c|c|c|c|c|c|c|c|c|c|c|c|c|c|}
\hline \multirow[b]{2}{*}{ Cycle No. } & \multicolumn{14}{|c|}{$A$} \\
\hline & 1 & 2 & 3 & 4 & 5 & 6 & 7 & 8 & 9 & 10 & 11 & 12 & 13 & 14 \\
\hline & $v$ & $\mathrm{R}$ & $v$ & A & I & $\mathrm{N}$ & G & $\mathrm{F}$ & G & $\mathbf{R}$ & I & G & $\mathrm{R}$ & L \\
\hline & $P$ & $F$ & I & $\begin{array}{l}\text { T } \\
\text { B }\end{array}$ & $\mathrm{L}_{\mathrm{L}}^{\mathrm{N}}$ & D & Y & A & A & Y & M & $\mathrm{F}$ & K & Y \\
\hline & $p$ & A & $N$ & L. & $P$ & W & $\begin{array}{l}\text { G } \\
S\end{array}$ & $S$ & $\begin{array}{l}S \\
G\end{array}$ & $\begin{array}{l}N \\
D\end{array}$ & $\begin{array}{l}y \\
S\end{array}$ & $\begin{array}{l}D \\
V\end{array}$ & I & A \\
\hline & & & & & & & & B & & & & & & \\
\hline \multirow[t]{4}{*}{ Cycle No. } & 1 & 2 & 3 & 4 & 5 & 6 & 7 & 8 & 9 & 10 & 11 & 12 & 13 & 14 \\
\hline & $v$ & $R$ & $V$ & A & I & $\mathrm{N}$ & G & $F$ & G & 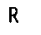 & I & G & $\mathrm{R}$ & L \\
\hline & $P$ & $F$ & I & $T$ & $\mathrm{~N}$ & D & $Y$ & A & A & Y & M & $F$ & k & Y \\
\hline & $P$ & A & $\mathrm{N}$ & $L$ & $P$ & $W$ & G & $S$ & $S$ & $\mathrm{~N}$ & $v$ & $D$ & 1 & A \\
\hline
\end{tabular}

Figure 2. Sequences of GAPDH peptides after formic acid treatment.

A. Expected from sequence determinations already published. B. Determined in the present work. Nomenclature as in Figure 1. 
Attempts to fractionate the peptides obtained by the cyanogen bromide cleavage of GAPDH on both Biogel P-10 and P-30 were not completely successful. However, sufficient separation was obtained which enabled the sequence identification of residues 133 to 136 as -AsnGlu-Val-Lys- and of residue 236 as aspartic acid.

When a cyanogen bromide cleavage of GAPDH was subjected to trypsin treatment, one peptide was obtained (residues 269 to 303) which is twice as long as any other peptide produced by this combination of chemical and enzymatic treatments. Chromatographic separation of the peptide mixture (see section 2.3.2.1.) and sequence analysis of some of the peptide peaks indicated that residues 275 and 276 were glutamic and aspartic acids, respectively. In addition, residues 173 to 178 could be identified as -Thr-Thr-Val-His-Ser-Leù- and residues 312 to 314 were -Asp-Asn-Glu-

Treatment of proteins with o-iodosobenzoic acid has been recently shown (6) to hydrolyse tryptophanyl bonds in proteins in high yields. Since GAPDH contains only three tryptophan residues, two of which occur at inconsistent sequence stretches, this reagent would appear to be of excellent use in the present studies. Fractionation of the resulting peptides gave five well-defined peaks absorbing at $280 \mathrm{~nm}$ which were subjected to sequence analysis. It was found, however, that tyrosyl bonds were also hydrolysed to varying degrees, under the conditions used in these experiments. Sequence analyses of the peptides confirmed the previous assignment of residues 275 as glutamic acid, identified residues 276,282 , and 286 as aspartic acids, and residue 287 as serine. In addition residues 85 to 93 were identified as

-Gly-Ser-Ser-Asn-Val-Asp-Ile-Ala-Ile-

Since in the genetic code, a single base change is required to alter a tyrosine to a tryptophan triplet, both ribonuclease and yeast superoxide dismutase which contain no tryptophans but six tyrosines and one tyrosine, respectively, were treated with o-iodosobenzoic acid as described for GAPDH. Subsequent sequence analysis indicated that tyrosyl bonds had been hydrolysed in these two proteins, but as in the case of GAPDH, the cleavage was not complete. Thus, it would appear that the original total of three tryptophan residues for GAPDH as found by amino acid analysis and recorded in the present sequence is correct.

Carboxypeptidase $\mathrm{Y}$ treatment of the whole protein indicated that residue 328 was isoleucine, in agreement with that predicted from the gene sequence.

In the present communication we have described the identification of 40 residues of 47 inconsistent amino acid sequence assignments in the primary structure of GAPDH. Of the seven remaining undetermined residues, residue 293 can either be an asparagine or aspartic acid and residue 299 is either glutamic acid or glutamine; the remaining five residues show no variation within the three previously published sequences. In summary, our results agree with those predicted by the gene structure and it appears safe to assume that the seven amino acids remaining to be firmly established by sequence analysis of the protein are indeed those predicted from the gene structure. Our results demonstrate indirectly that nucleotide sequencing can be reliable at least in those cases where a partial amino acid sequence has previously been established.

\section{ACKNOWLEDGEMENTS}

The authors wish to thank drs. M. OTTESEN and T. Viswanatha for helpful discussion and mrss. Lone Sørensen and Bodil Corneliussen for skilled technical assistance.

\section{REFERENCES}

1. Harris, J. I. \& M. Waters: Glyceraldehyde-3phosphate dehydrogenase. In: The Enzymes XIII, 1-49 (1976)

2. Holland, J. P. \& M. J. Holland: The primary structure of a glyceraldehyde-3-phosphate dehydrogenase gene from Saccharomyces cerevisiae. J. Biol. Chem. 254, 9839-9845 (1979)

3. Johansen, J. T., C. Overballe-Petersen, B. Martin, V. Hasemann \& I. Svendsen: The complete amino acid sequence of copper.zinc superoxide dismutase from Saccharomyces cerevisiae. Carlsberg Res. Commun. 44, 201-217 (1979)

4. Jones, G. M. T. \& J. I. Harris: Glyceraldehyde-3-phosphate dehydrogenase: amino acid sequence of enzyme from baker's yeast. FEBS Lett. 22, 185-189 (1972) 
5. LANDON, M.: Cleavage at aspartyl-prolyl bonds. In: Methods in Enzymology 47, 145-149 (1977)

6. Mahoney, W. C. \& M. A. Hermodson: Highyield cleavage of tryptophanyl peptide bonds by o-iodosobenzoic acid. Biochemistry 18, 38103814 (1979)
7. Stallcup, W. B., S. C. Mockrin \& D. E. KoshLAND: A rapid purification procedure for glyceraldehyde-3-phosphate dehydrogenase from baker's yeast. J. Biol. Chem. 247, 6277-6279 (1972) 